or all these members $B$ is variable with temperature. It probably follows that for all liquids $B$ is a function of temperature, and that the difference between associated and non-associated liquids with regard to $B$ is entirely a matter of degree.

For other families of compounds similar lines exist, but a better rendering of these curves is obtained by plotting the function $\eta_{B} / \eta_{i}$ against $T / T_{B}$, as shown in Fig. 2.

The results of 137 liquids have been plotted representing $n$-paraffins (12), iso-paraffins (15), acyclic olefines (7), cyclo-hexene, alkyl halides (25), alcohols (11), acetaldehyde, ketones (4), anhydrides (2), diethyl-ether, carboxylic acids (5), water, cyclic compounds (30), elements (12) and inorganic compounds (10), resulting in the following conclusions :

(1) All the normal or unassociated liquids fall in a fan-shaped manner in the shaded portion of the graph. In this area, also, fall such compounds as the carboxylic acids which are known not to change appreciably the degree of association on rise of temperature and in fact evaporate to a great extent in the dimer form. Thus the shaded portion contains all compounds which are either unassociated, or if associated, have a small coefficient for the increase of association with a fall in temperature.

(2) The upper portion of the graph contains associated liquids. It is remarkable that water changes its degree of association very slowly, as the evidence of the plot tends to show, when compared with trimethyl carbinol.

(3) The lower portion of the graph is characteristic of liquid metals. Mercury shows the regularity of the points on the curve.

A full description of the work and the results obtained has been prepared for publication and will appear in the literature later in this year.

A. H. Nissan.

L. V. W. Clark.

Department of Oil Engineering and Refining,

University,

Birmingham.

${ }^{1}$ Thorpe and Rodger, Phil. Trans., A, 185, 397 (1894).

${ }^{2}$ Bauer, Magat and Surdin, Trans. Faraday Soc., 33, 81 (1937).

\section{Surface Tension and Lindemann Frequency}

ANDRADE $^{1}$ has derived the expression $\eta=\frac{4}{3} \frac{m \nu}{\sigma}$ for the viscosity $(\eta)$ of a liquid at its melting point in terms of the molecular mass $(m)$, the Lindemann frequency $(\nu)$ and the mean molecular distance $(\sigma)$. This simple relation has been amply substantiated by experimental results and has given us an insight into the mechanism of viscosity in liquids. But so far as we are aware, no equally elegant explanation of the phenomenon of surface tension in liquids has been put forward.

Let us assume that at the melting point the energy per unit area of the surface is given by the total energy of the molecules in a surface layer of unit area, each molecule vibrating with a frequency equal to the Lindemann frequency. Considering that the oscillation is simple harmonic with an amplitude $a$, it follows on the basis of the assumptions that the surface tension

$$
\gamma=\Sigma \frac{1}{2} m a^{2}(2 \pi \nu)^{2}=\frac{2 \pi^{2} a^{2}}{\sigma^{2}} m \nu^{2} .
$$

Assuming, after Lindemann and others, that at the melting point $a / \sigma$ is constant, we obtain

$$
\nu=k \sqrt{\frac{\gamma}{m}}
$$

a relation dimensionally correct. Calculating the Lindemann frequency both for elements and compounds from the relation $v=2.80 \times 10^{12} \sqrt{T_{8} / M V^{2 / 3}}$ where $T_{8}$ is the melting point, and $M$ and $V$ refer to the atomic weight and the atomic volume respectively in the case of elements, and to the molecular weight and the molecular volume respectively in the case of compounds ${ }^{2}$, and dividing the frequency so obtained by $\sqrt{\gamma / m}$, we find that the value of $k$ in the case of forty-six substances examined, of which twenty-four are elements, ranges between 1 and $3 \cdot 5$, with a mean value of $2 \cdot 34$. The variation of surface tension with temperature can thus be explained on the same lines as that of viscosity.

Details will be published elsewhere.

L. Sibaiya.

M. Rama Rao.

University of Mysore, Central College, Bangalore. Feb. 22.

${ }^{1}$ Andrade, Phil. Mag., 17, 497-511 (1934).

${ }^{2}$ Sibaiya and Rama Rao, Cur. Sci., 8, 12 (1939).

\section{Influence of Electrical Conductivity of Surroundings on the Natural Wave-length of an Antenna}

THE free end of an antenna fed by an oscillator is connected to a metal plate under which is placed a basin of water. The conductivity of the water is changed by adding known weights of various salts. The solution is earthed and the distance between the plate and the solution is kept constant.

It has been found that the natural wave-length of the antenna increases with this conductivity up to a definite limit and then becomes constant for greater concentrations. This definite concentration marks the limit between the dielectric and the conductive state of the solution. The increase of the natural wave-length for small concentrations can be attributed to the fact that the dielectric constant of the solution increases with concentration.

Experiments are now being carried out for different wave-lengths and different electrolytes. The effect of this conductivity on the resistance of the antenna is also being investigated.

$$
\begin{gathered}
\text { Physics Department, } \\
\text { Egyptian University, } \\
\text { Cairo. }
\end{gathered}
$$

\section{R. NAKHLA.}

\section{Hydrolytic De-amination of Amino-Acids}

Virtanen and Erkama ${ }^{1}$ discovered recently in B. fluorescens liquefaciens an enzyme which causes hydrolysis of aspartic acid into malic acid and ammonia. This, however, is not the first example of hydrolytic de-amination of amino-acids. In a series of papers ${ }^{2}$, we have been able to show that in aqueous solution, amino-acids are hydrolysed under the 